\title{
7 Managementprozesse und Finanzierung des Berliner TransitionsProgramms
}

\subsection{Einleitung}

Das Berliner TransitionsProgramm ist ein indikationsübergreifendes Betreuungsprogramm für Jugendliche und junge Erwachsene mit chronischen Erkrankungen. Das Programm ersetzt keine diagnostischen und therapeutischen Leistungen der Regelversorgung, sondern ergänzt die medizinische Behandlung um notwendige Informations- und Koordinationsleistungen, die in der Versorgungsrealität bislang unzureichend berücksichtigt werden. Bei dem BTP handelt es sich um ein auf verschiedene Patientengruppen übertragbares Interventions- und Rahmenkonzept für die Transitionsphase, das mit Kostenträgern im Gesundheitswesen verhandelt und als definierter Behandlungs- und Betreuungskomplex in der Versorgung etabliert werden kann. Das Interventions- und Rahmenkonzept (vgl. ausführlich Abschnitt 6) umfasst Vorgaben zur Struktur- und Prozessqualität, die unter Berücksichtigung des aktuellen Standes diagnostischer und therapeutischer Standards in dem jeweiligen Indikationsgebiet sowie der Transitionsmedizin an die Erfordernisse verschiedener Patientengruppen und an unterschiedliche regionale Versorgungsstrukturen angepasst werden können.

Allgemein gesprochen ist für die Durchführung des Programms die Einrichtung einer „Transitionsstelle“ erforderlich, die im Wesentlichen folgende Aufgaben übernimmt:

- Regionale Organisation und Weiterentwicklung des Programms (inkl. z. B. Öffentlichkeitsarbeit),

- Entwicklung des Netzwerks und Vertragsabschluss mit Leistungserbringern in Pädiatrie und Erwachsenenmedizin, die programmspezifische zusätzliche Leistungen erbringen,

- Verhandlung und Abschluss von Verträgen mit Kostenträgern,

- Abrechnung der vereinbarten Zusatzleistungen mit den vertraglich verbundenen Leistungserbringern,

- Abrechnung der mit den Kostenträgern für die Programmteilnahme ihrer Versicherten vereinbarten Vergütungen,

- Durchführung bzw. Sicherstellung des Fallmanagements.

Eine Transitionsstelle tritt somit nicht selber als Leistungserbringer im Sinne der Regelleistungen der gesundheitlichen Versorgung gemäß Sozialgesetzbuch SGBV auf, sondern nimmt als Managementgesellschaft eine koordinierende und steuernde Funktion einschließlich des Fallmanagements wahr. 
Die ursprüngliche Transitionsstelle des BTP ist an den DRK Kliniken BerlinWestend angesiedelt. In der Erprobungsphase (2009-2011) erstreckte sich das Einzugsgebiet zunächst nur auf Berlin und Brandenburg sowie die beiden Pilotindikationen Diabetes Typ 1 und Epilepsien. Inzwischen betreut die Transitionsstelle an den DRK Kliniken auch Patienten in anderen Bundesländern sowie mit weiteren Indikationen. Die Einzelheiten der regionalen Erstreckung sowie der erfassten Indikationen sind in den Verträgen zwischen den Kostenträgern und den DRK Kliniken Berlin als dem Träger der Transitionsstelle konkret festgelegt.

Das Berliner TransitionsProgramm (BTP) als Betreuungsprogramm ist unabhängig von der operativen Transitionsstelle an den DRK Kliniken Berlin zu sehen: Nach Abschluss der Entwicklungsphase wurde auf Initiative der Autoren des BTP 2014 der Verein „Berliner TransitionsProgramm e. V.“ (Amtsgericht Charlottenburg, VR 33830 B) gegründet. Dieser Verein soll künftig die Weiterentwicklung und Verbreitung des BTP betreiben sowie die Gründung weiterer Transitionsstellen in anderen Regionen unterstützen, die nach dem BTP-Konzept arbeiten wollen. Das vorliegende Handbuch ist ein wesentliches Instrument auf diesem Weg.

Die Ausführungen in diesem Kapitel sollen interessierten Initiativen Hinweise geben, wie eine nach dem Konzept des BTP arbeitende Transitionsstelle eingerichtet werden kann, welche Aufgaben zu erfüllen sind und welche Optionen für die Finanzierung eines regionalen Transitionsprogramms existieren.

Für die Planung einer Transitionsstelle wurde im Rahmen eines von der Robert Bosch Stiftung geförderten Folgeprojekts durch das IGES Institut ein umfangreiches auf MS-Excel basierendes Werkzeug zur „Geschäftsplanung Transitionsmanagement“ entwickelt, das eine detaillierte Planung und Berechnung aller relevanten Parameter erlaubt. Im Folgenden werden zunächst überblicksartig die wesentlichen Aspekte des Managements und der Finanzierung dargestellt. Im anschließenden Kapitel 8 wird die Planung eines Transitionsprogramms nach dem BTP-Konzept anhand der Funktionalitäten des Planungstools im Detail dargestellt.

\subsection{Managementprozesse}

Das durch eine Transitionsstelle zu leistende Management eines Transitionsprogramms nach dem BTP-Modell umfasst die in Tab. 7.1 dargestellten Ebenen.

\subsubsection{Fallmanagement}

Das BTP hat zum Ziel, dass die betroffenen Patienten nach dem Transfer in der Erwachsenenmedizin nachhaltig verankert sind und dort hinsichtlich ihrer krankheitsspezifischen Erfordernisse adäquat spezialisiert versorgt werden. Das Fallmanagement ist Ansprechpartner für die teilnehmenden Patienten und behandelnden Ärzte. Es 
Tab. 7.1: Managementleistungen im Berliner TransitionsProgramm.

\begin{tabular}{ll}
\hline Managementleistungen & Beschreibung \\
\hline Fallmanagement & - Planung/Umsetzung des individuellen Unterstützungs- \\
& bedarfes \\
& - Koordination der Transitionsmaßnahmen \\
\hline Netzwerkmanagement & - Kontaktierung, Information und Gewinnung geeigneter Leis- \\
& - tungserbringer als Kooperationspartner \\
\hline Finanzierung und Vergütung & Abschluss von Kooperationsverträgen mit Leistungserbringern \\
& - Abschluss von Verträgen mit Kostenträgern \\
& - Abrechnung mit den kooperierenden Leistungserbringern \\
& - Abrechnung mit den Kostenträgern \\
\hline Dokumentation und Evaluation & - Dokumentation im Rahmen von Fallmanagement und Quali- \\
& - tätssicherung \\
& Auswertungen und Berichte zur Evaluation \\
\hline
\end{tabular}

Quelle: Berliner TransitionsProgramm

plant und operationalisiert den individuellen Unterstützungsbedarf, koordiniert die Maßnahmen innerhalb des Transitionsprogramms, ist zuständig für die Dokumentation in der Patientendatenbank sowie für die Sammlung der zur Evaluation benötigten Daten. Nähere Einzelheiten zu den Leistungen des Fallmanagements sind in Kapitel 6.3.4 dargestellt.

Im Rahmen des Managements einer Transitionsstelle müssen geeignete Fallmanger gefunden und eingestellt sowie die erforderlichen räumlichen und technischen Infrastrukturen geschaffen werden. Die Kompetenzprofile von Fallmanagern sind in Kapitel 6.3.4 dargestellt. Darüber hinaus sind in den aktuellen Verträgen der Transitionsstelle an den DRK Kliniken Berlin mit den Kostenträgern auch formale Anforderungen an die Fallmanager festgelegt:

Für den Einsatz als Fallmanager/in im Rahmen des Transitionsprogramms kommen Personen mit einem heilkundlichen oder gesundheitsnahen Berufsabschluss sowie einer Zusatzqualifikation im Bereich des Fallmanagements in Frage. Diese Zusatzqualifikation soll entweder durch einen von der Deutschen Gesellschaft für Care und Case Management (DGCC) zertifizierten Weiterbildungsabschluss oder durch eine vergleichbare akademische Weiterbildung, z. B. im Bereich Pflegewissenschaften/Pflegemanagement, nachgewiesen werden.

Die Berechnung der erforderlichen personellen Kapazitäten für das Fallmanagement ist Teil des Planungstools und wird in Kapitel 8 erläutert.

Im Hinblick auf die erforderlichen Infrastrukturen ist - neben der üblichen Ausstattung mit Räumen, IT und Bürokommunikation - vor allem der Einsatz einer speziellen Fallmanagement-Software zu thematisieren. In Kapitel 6.3.5 sind die Funktio- 
nalitäten des von der Transitionsstelle in den DRK Kliniken Berlin eingesetzten Programms beschrieben.

Die Arbeit der Fallmanager innerhalb eines Transitionsprogramms muss zur Qualitätssicherung mit den übrigen Programmbeteiligten - insbesondere den Verantwortlichen für das Gesamtprogramm und das Netzwerkmanagement - regelmäßig rückgekoppelt werden. In der Transitionsstelle an den DRK Kliniken Berlin-Westend sind regelmäßige Teambesprechungen etabliert und die Durchführung von Qualitätszirkeln unter Nutzung der Falldokumentation geplant.

\subsubsection{Netzwerkmanagement}

Das Netzwerkmanagement beinhaltet den Aufbau und die Pflege tragfähiger Beziehungen zu den zuweisenden Kinder- und Jugendspezialisten sowie den weiterbehandelnden Ärzten in der Erwachsenenmedizin. Das Management der Transitionsstelle schließt die erforderlichen Kooperationsverträge und weist die Leistungserbringer in die transitionsspezifischen Leistungen ein. Diese Leistungen (Transitionsgespräche, Epikrisen usw.) sind in Kapitel 6.3 im Detail dargestellt.

Für die Funktionsfähigkeit eines regionalen Transitionsprogramms ebenso wie für die wirtschaftliche Lebensfähigkeit einer Transitionsstelle ist das Netzwerk der Kooperationspartner von entscheidender Bedeutung: Innerhalb des Einzugsgebiets der Transitionsstelle müssen auf der „pädiatrischen Seite“ möglichst alle Praxen und Zentren als Kooperationspartner gewonnen werden, die die Patienten mit den erfassten Indikationen behandeln. Das zu erwartende Aufkommen von Programmteilnehmern in einer Region wird neben der Epidemiologie vor allem durch die Kooperationsbereitschaft der Versorgungseinrichtungen auf der „pädiatrischen Seite“ determiniert.

Der Aufbau des Netzwerks von Kooperationspartnern erfordert vor allem persönliche Gespräche mit den verantwortlichen Ärzten. Im Rahmen der Entwicklung des BTP bzw. des Aufbaus der Transitionsstelle an den DRK Kliniken Berlin-Westend wurden zahlreiche Vorträge bei regionalen Fortbildungsveranstaltungen der einzelnen Fachgruppen und Disziplinen gehalten und Gespräche mit einzelnen Ärzten in großer Zahl geführt. Sobald das Transitionsprogramm in der Region einen gewissen Bekanntheitsgrad erreicht hat, ist erfahrungsgemäß auch mit entsprechenden Einladungen zu rechnen, da sich nahezu alle pädiatrischen Subdisziplinen um Lösungen für die Transitionsproblematik bei ihren jeweiligen Patienten bemühen. Da die absoluten jährlichen Zahlen von Transitionspatienten auch in großen pädiatrischen Zentren relativ klein sind, besteht häufig Interesse an einer Bündelung dieser Aufgabe in einem indikationsübergreifenden regionalen Transitionsprogramm.

Neben dem pädiatrischen Netzwerk für den „Zufluss“ von Patienten muss parallel für den „Abfluss“ ein Netzwerk im Bereich der jeweils relevanten Fachrichtungen der Erwachsenenmedizin aufgebaut werden. Auch sind Vorstellungen des Programms im Rahmen von Fortbildungsveranstaltungen der jeweiligen Fächer zu empfehlen. 
Darüber hinaus spielen in diesem Kontext persönliche Empfehlungen und Kontakte erfahrungsgemäß eine besonders wichtige Rolle: Die pädiatrischen Zentren können meistens Kollegen in der Erwachsenenmedizin nennen, an die sie häufig Patienten verweisen bzw. zu denen sie gute Kooperationsbeziehungen unterhalten.

$\mathrm{Zu}$ den Aufgaben des Managements einer Transitionsstelle gehört der Abschluss von Kooperationsverträgen sowohl mit den pädiatrischen als auch mit den erwachsenenmedizinischen Partnern. Diese Verträge sowie die resultierenden Verzeichnisse der Kooperationspartner sind auch Bestandteil des Vertragswerks zwischen der Transitionsstelle und den Kostenträgern.

Die Kooperationspartner müssen bei Vertragsabschluss alle erforderlichen Informationen und Dokumente über die Programmdurchführung, die vereinbarten programmspezifischen Leistungen und die von ihnen zu führenden Dokumentationen erhalten. Ferner sind in den Kooperationsverträgen die Honorare für programmspezifische Leistungen und die Abrechnungsbestimmungen aufgeführt. Die Struktur der im BTP verwendeten Kooperationsverträge zeigt Tab. 7.2.

\subsubsection{Finanzierung und Vergütung}

Die Finanzierung des BTP erstreckt sich ausschließlich auf Leistungen und Aufgaben, die nicht Bestandteil der Regelversorgung im Rahmen der Gesetzlichen Krankenversicherung sind, sondern die als Teil des Programms zusätzlich erbracht werden.

Dabei handelt es sich um folgende vier Komponenten:

1. Vergütungen, die die ärztlichen Kooperationspartner des BTP für die Erbringung transitionsspezifischer Leistungen erhalten,

2. Finanzierung des Fallmanagements,

3. Finanzierung der Aktivitäten zur Programmpflege sowie zum Auf- und Ausbau der Netzwerke von Vertrags- und Kooperationspartnern,

4. administrative Kosten.

In den folgenden Abschnitten werden die Grundlagen der Kalkulation dieser vier Komponenten erläutert. Die Vergütung des BTP erfolgt über fallbezogene Pauschalen, d.h., für jeden am BTP teilnehmenden Patienten wird den Kostenträgern eine zuvor vereinbarte Fallpauschale in Rechnung gestellt. Diese Pauschale deckt die kalkulierten Kosten aller vier Komponenten ab.

Die Ableitung eine solchen Fallpauschale ist eine wesentliche Funktion des Geschäftsplanungstools (vgl. Kapitel 8). 
Tab. 7.2: Struktur der Kooperationsverträge des BTP mit den kooperierenden Ärzten (Kinder- und Jugendspezialisten und Erwachsenenspezialisten).

\begin{tabular}{|c|c|}
\hline Abschnitt & Inhalte \\
\hline $\begin{array}{l}\text { Angaben zum Vertrags- } \\
\text { partner }\end{array}$ & Name, Anschrift, Rechtsform \\
\hline $\begin{array}{l}\text { Angaben zur Zulassung und } \\
\text { Qualifikation des Vertrags- } \\
\text { partners }\end{array}$ & $\begin{array}{l}\text { Gebietsbezeichnung, Schwerpunkte/Zusatzbezeichnungen, } \\
\text { Lebenslange Arztnummer, Betriebsstättennummer }\end{array}$ \\
\hline Erklärung der Mitwirkung & $\begin{array}{l}\text { Die [Name der Transitionsstelle] hat mich bzw. die von mir vertre- } \\
\text { tene Institution im Rahmen des [Bezeichnung Vertrag zwischen } \\
\text { Transitionsstelle und Kostenträgern/Bezeichnung des Programms] } \\
\text { zur Erbringung ambulanter ärztlicher Leistungen in den folgenden } \\
\text { Indikationen eingebunden [Indikationen angeben]. Die Verträge } \\
\text { der jeweiligen Kostenträger sowie die relevanten Anlagen [Auf- } \\
\text { führung der relevanten Dokumente aus dem Vertrag zwischen } \\
\text { Transitionsstelle und Kostenträgern, z. B. Leistungsbeschreibung, } \\
\text { Verzeichnis der Materialien, Patienteninformation usw.] wurden } \\
\text { mir ausgehändigt. Ich verpflichte mich, über die Vertragsinhalte } \\
\text { Stillschweigen zu bewahren und die zur Verfügung gestellten } \\
\text { Dokumente nicht an Dritte weiterzugeben. Über meine Pflichten } \\
\text { im Programm wurde ich informiert. }\end{array}$ \\
\hline Vergütungsvereinbarung & $\begin{array}{l}\text { Für die Leistungserbringung wurden die in dieser Vereinbarung } \\
\text { aufgeführten Vergütungen vereinbart. }\end{array}$ \\
\hline $\begin{array}{l}\text { Zustimmung zur Nennung } \\
\text { des Kooperationspartners im } \\
\text { Kontext der Öffentlichkeitsar- } \\
\text { beit für das Programm }\end{array}$ & $\begin{array}{l}\text { Ich bin damit einverstanden, dass Name und Adressdaten von mir } \\
\text { bzw. der von mir vertretenen Institution zu Informationszwecken } \\
\text { über das Programm, insbesondere auch im Internet-Auftritt der } \\
\text { Krankenkassen, genutzt werden können. }\end{array}$ \\
\hline $\begin{array}{l}\text { Verpflichtungserklärung } \\
\text { und Unterschrift }\end{array}$ & $\begin{array}{l}\text { Hiermit verpflichte ich mich bzw. die von mir vertretene Institution, } \\
\text { die im Rahmen des Vertrags vereinbarten Leistungen zu erbringen } \\
\text { und die Bestimmungen zur Geheimhaltung und zum Datenschutz } \\
\text { einzuhalten. }\end{array}$ \\
\hline
\end{tabular}

Quelle: Berliner TransitionsProgramm

\section{Vergütungen für programmspezifische Zusatzleistungen der ärztlichen Kooperationspartner}

Die kooperierenden ärztlichen Leistungserbringer werden für folgende Leistungen durch das BTP vergütet:

\section{Kinder-/Jugendspezialisten:}

- erstes Transitionsgespräch, einschließlich Dokumentation und Informationsübermittlung an das Fallmanagement (vor Transfer des Patienten),

- Erstellung und Übermittlung der Strukturierten Epikrise. 
Erwachsenenspezialisten:

- zweites Transitionsgespräch, einschließlich Dokumentation und Informationsübermittlung an das Fallmanagement (nach erfolgtem Transfer des Patienten),

- Abschlussgespräch, einschließlich Dokumentation und Informationsübermittlung an das Fallmanagement.

Beide Gruppen von Kooperationspartnern:

- optionale Durchführung des zweiten Transitionsgesprächs als gemeinsame Sprechstunde von Kinder-/Jugend- und Erwachsenenspezialist,

- optionale Durchführung einer gemeinsamen Fallkonferenz.

Diese Leistungen werden unter Berücksichtigung des erforderlichen Zeitaufwands für die Ärzte und unter Zugrundelegung üblicher Kostensätze je Zeiteinheit vergütet. Die Abrechnung dieser Honorare erfolgt zwischen der Transitionsstelle und den Kooperationspartnern.

\section{Finanzierung des Fallmanagements}

Für das Fallmanagement ist eine Vergütung nach Art einer Einzelleistung nicht praktikabel, weil die Fallmanager neben der regulären Begleitung der eingeschriebenen Programmteilnehmer auch zahlreiche Beratungsgespräche mit Patienten und Eltern führen, die nicht in eine Programmteilnahme münden. Diese Beratungs- und Informationsfunktion ist ausdrücklicher Bestandteil des Programms, d. h., die ärztlichen Kooperationspartner des BTP informieren zunächst ihre Patienten der betreffenden Altersgruppen regelhaft über das BTP. Viele dieser Patienten oder deren Eltern melden sich dann telefonisch bei den Fallmanagern mit der Bitte um Informationsmaterial bzw. ein Beratungsgespräch. Bei der Mehrzahl reichen ein Informationsgespräch und die Zusendung der BTP-Broschüre als Unterstützung aus. Nur bei Hinweisen auf einen größeren Unterstützungsbedarf bzw. auf ausdrückliche Empfehlung des Kinder- und Jugendspezialisten wird zu einer Programmteilnahme geraten.

Bei der Finanzierung des Fallmanagements im BTP wird zum einen dieser Aufwand für die Information und Beratung berücksichtigt, zum anderen wird der Heterogenität der Teilnehmer Rechnung getragen: Der Aufwand für das Fallmanagement kann je nach Situation des Patienten stark variieren und reicht von kurzen telefonischen Beratungen bis zu häufigen Kontaktaufnahmen und intensiven Gesprächen mit den Fallmanagern.

Für die Vergütung des Fallmanagements wurden daher zunächst drei Falltypen mit unterschiedlichem durchschnittlichen Aufwand definiert:

- Typ 1: nur Beratung und Informationsübermittlung, aber keine Transitionsbegleitung,

- Typ 2: reguläre Transitionsbegleitung,

- Typ 3: komplexe Transitionsbegleitung. 
Für jeden Falltyp wurde auf Grundlage der Erfahrungen aus dem Erprobungsprojekt der Zeitaufwand kalkuliert und die Auftretenshäufigkeit der drei Typen ermittelt. Auf dieser Grundlage wurde berechnet, welches Gesamtfallvolumen (alle drei Falltypen zusammen) ein Fallmanager bei einer Vollzeitstelle bewältigen kann. Gegenüber den Kostenträgern tatsächlich abgerechnet werden nur die Patienten der Typen 2 und 3, da lediglich diese Fälle tatsächlich an dem regulären Programmablauf teilnehmen. Die Kosten für Typ 1 sind auf diese Fälle der Typen 2 und 3 umgelegt.

Entscheidend für den Finanzierungsbedarf des Fallmanagements sind - neben den Personalkosten für die Fallmanager - somit die Zeitaufwände für die Begleitung der drei Falltypen sowie deren Häufigkeiten.

In dem Planungstool lassen sich alle genannten Parameter einstellen und damit der Finanzierungsbedarf für das Fallmanagement berechnen (vgl. unten).

\section{Finanzierung der Aktivitäten zur Programmpflege sowie zum Auf- und Ausbau der Netzwerke von Vertrags- und Kooperationspartnern}

Die erforderlichen Aktivitäten zum Aufbau eines Netzwerks von Kooperationspartnern sind oben bereits dargestellt worden. Darüber hinaus sind durch die Transitionsstelle die Weiterentwicklung bzw. Anpassung des Programms selbst, d. h. der programmspezifischen Leistungen, der Dokumente und Materialien, sowie das kontinuierliche Qualitätsmanagement zu leisten.

Die Aufwände bzw. der Finanzierungsbedarf für diesen Aufgabenkomplex hängen einerseits vom Zuschnitt des Programms (Einzugsgebiet, erfasste Indikationen) und andererseits von den Personen ab, die diese Aufgaben wahrnehmen. Vor allem die beschriebenen Aktivitäten des Netzwerkaufbaus müssen zu einem nennenswerten Teil durch Ärzte wahrgenommen werden. In den vom BTP bzw. von der Berliner Transitionsstelle mit den Kostenträgern geschlossenen Verträgen ist dafür ein auf Jahresbasis kalkuliertes Stundenbudget für die am BTP beteiligten Ärzte der Transitionsstelle vorgesehen. Das entsprechende Finanzvolumen ist auf die fallweise mit den Kostenträgern abgerechneten Pauschalen umgelegt.

\section{Administrative Kosten}

Als administrative Kosten einer Transitionsstelle sind vor allem zu kalkulieren:

- Raumkosten (Miete inkl. Nebenkosten) für die Transitionsstelle (insbesondere Arbeitsplätze des Fallmanagements),

- technische Ausstattung (Hardware), die Kosten werden im Geschäftsplanungstool unter Berücksichtigung der Abschreibungszeiträume als monatliche Kosten kalkuliert,

- Softwarelizenzen, Gebühren (Telefon, Internet usw.),

- Büromaterial, Porto, Druckkosten.

Diese fixen Kosten werden ebenfalls auf die Fallpauschale umgelegt. 


\section{Vergůtung über eine Fallpauschale}

Die Programmkosten werden über eine patientenbezogene Pauschale mit den Kostenträgern abgerechnet. Das heißt, die Krankenkassen zahlen einmalig pro teilnehmendem Versicherten über die gesamte Laufzeit des Transitionsprozesses eine Fallpauschale, deren Höhe im Vorhinein zu kalkulieren und mit den Kostenträgern zu verhandeln ist. Die Pauschale setzt sich aus den vier beschriebenen Komponenten zusammen. Dabei ist vor allem zu berücksichtigen, dass nur für die tatsächlich teilnehmenden Versicherten eine Pauschale gezahlt wird, die folglich in ihrer Höhe so bemessen sein muss, dass auch die fixen Kosten (Netzwerkmanagement, administrative Kosten) sowie die für eine einzelfallabhängige Vergütung nicht geeigneten Leistungen (insbes. Beratungsgespräche des Fallmanagements, die nicht in eine Programmteilnahme münden) finanziert werden können.

Mit dem Geschäftsplanungstool lässt sich, ausgehend von den individuellen Daten der geplanten Transitionsstelle, die Höhe einer solchen Pauschale kalkulieren.

Die gesamte Auszahlung bzw. Fakturierung der Pauschale erfolgt im BTP je nach Vertrag mit den Kostenträgern nicht in einem Schritt, sondern geknüpft an definierte Meilensteine, die der teilnehmende Patient im Transitionsprozess erreicht.

\subsubsection{Dokumentation und Evaluation}

Im BTP wird unter Nutzung eines speziellen Software-Programms eine patientenbezogene Dokumentation des Transitionsprozesses geführt (vgl. dazu Kapitel 6.3.5). Auf Basis dieser Dokumentation werden ferner die von den Kostenträgern verlangten Berichte zur Evaluation und Qualitätsdokumentation erzeugt.

In den Verträgen mit den Kostenträgern sind unterschiedliche Regelungen zur Evaluation des Programms getroffen. Grundsätzlich kommen folgende Maßnahmen für eine laufende Evaluation und Berichterstattung in Betracht:

- zusammenfassende Berichte über den Programmdurchlauf und das Erreichen definierter Meilensteine (z. B. Zahl der Kontakte mit dem Fallmanagement vor/nach Transfer; durchschnittliches Zeitintervall zwischen Programmeintritt und Transfer; Anteil Patienten, der nach einem Jahr noch beim zuerst gewählten Erwachsenenspezialisten in kontinuierlicher Behandlung ist; usw.),

- begleitende Patientenbefragungen (z. B. unter Nutzung der Fragebögen, die in der Erprobungsphase des BTP entwickelt und eingesetzt wurden),

- Analysen der Kostenträger auf Basis ihrer eigenen Daten, z. B. zu potenziell vermeidbaren Leistungsinanspruchnahmen in der Transitionsphase (z. B. Krankenhausaufnahmen, Notfallbehandlungen) oder zu anderen spezifischen Zielgrößen (z.B. Kontinuität der erforderlichen Arzneimittelverordnungen). 
Art und Umfang der von der Transitionsstelle zu Evaluationszwecken zu erhebenden Daten bzw. zu liefernden Berichte müssen individuell mit den Kostenträgern abgestimmt werden. Dabei müssen selbstverständlich die Regelungen des Daten- und Patientenschutzes eingehalten werden. Gegebenenfalls müssen die Patienten zusammen mit der Teilnahmeerklärung auch über die Erhebung und Nutzung von Daten aufgeklärt und ihr schriftliches Einverständnis dazu eingeholt werden. 